\title{
Fully Dynamic 3-Dimensional Orthogonal Graph Drawing*
}

\author{
M. Closson, S. Gartshore, J. Johansen, and S. K. Wismath ${ }^{1}$ \\ Department of Mathematics and Computer Science, \\ University of Lethbridge, \\ Lethbridge, Alberta, T1K-3M4, Canada. \\ wismath@cs.uleth.ca
}

\begin{abstract}
In a 3-dimensional orthogonal drawing of a graph, vertices are mapped to grid points on an integer lattice and edges are routed along integer grid lines. In this paper, we present a layout scheme that draws any graph with $n$ vertices of maximum degree 6 , using at most 6 bends per edge and in a volume of $O\left(n^{2}\right)$. The advantage of our strategy over other drawing methods is that our method is fully dynamic, allowing both insertion and deletion of vertices and edges, while maintaining the volume and bend bounds. The drawing can be obtained in $O(n)$ time and insertions/deletions can be performed in $O(1)$ time. Multiple edges and self loops are permitted. A more elaborate construction that uses only 5 bends per edge, and a simpler, more balanced layout that requires at most 7 bends per edge are also described.
\end{abstract}

\section{Introduction and Previous Work}

In this paper we describe a 3-dimensional orthogonal layout strategy for graphs of maximum degree 6 . In the final layout, vertices and edges lie on a 3-dimensional integer grid; in particular, vertices occur at grid points of the form $\left(x^{\prime}, y^{\prime}, z^{\prime}\right)$, where $x^{\prime}, y^{\prime}, z^{\prime} \in Z$, and edges are routed along integer-valued grid lines, possibly bending at integer grid points. Each edge $(u, v)$ joins the points representing vertices $u$ and $v$ and intersects no other vertex point. No pair of edges intersect (except at endpoints).

There have been several 3-dimensional layout strategies proposed in the graph drawing literature for graphs of maximum degree 6 - see [1], [3], [4], [5], [6]. Drawings of graphs of higher degree have been investigated in [2] and [5]. For the most part, these results have focussed on the trade-offs among: overall volume of the resulting layout, the maximum number of bends on an edge, the maximum length of an edge, etc. Experimental comparisons can be found in the paper by Di Battista, Patrignani, and Vargiu [3].

Although there are some classes of graphs of maximum degree 6 that can be drawn with few bends (e.g. trees can be drawn with 0 bends), the introduction of multiple edges, or self loops immediately implies that three bends per edge

* N.S.E.R.C. is gratefully thanked for financial assistance. 
may be required. For a self loop, the necessity of three bends is evident. A simple but tedious case study can be used to demonstrate that a pair of vertices with 6 edges between them, can not be drawn with at most two bends per edge.

Our result is motivated primarily by a dynamic version of the layout problem in which edges and vertices of the graph can be inserted or deleted over time. Papakostas and Tollis [5] introduced a semi-dynamic solution in which only vertices are permitted to be inserted and at all stages, the resulting layout must represent a connected graph. Our layout strategy is fully dynamic, allowing insertion and deletion of vertices and edges. The volume of the layout is bounded by $O\left(n^{2}\right)$, each edge has at most 6 bends, and insertions and deletions are accomplished in $O(1)$ time. The dynamic nature of our layout relies on the fact that at any time, a free port on any vertex may safely be connected to a free port of any other vertex without disturbing the layout, and the connection requires at most 6 bends.

Multiple edges and self-loops are also accommodated in our drawings. Multiple edges may prove to be an important consideration in applications that require some degree of "fault-tolerance". Previous 3-dimensional orthogonal constructions have typically been restricted to simple graphs and can not support dynamic operations. For example, one of the classic drawing algorithms in this area is due to Eades, Symvonis and Whitesides [4]. Their algorithm achieves $O\left(n^{1.5}\right)$ volume but uses 7 bends, requires a matching and colouring pre-processing step, and is only suitable in a static context. The same general technique has recently been modified by the authors to examine volume/bend tradeoffs and in particular they achieve a layout with at most 6 bends per edge and $O\left(n^{2}\right)$ volume.

One final feature of our layout that may be useful in some applications, is that the width of the layout is constant - in fact the entire drawing lies on 7 planes between the planes $Y=-3$ and $Y=+3$.

\section{Drawing Graphs in $O\left(n^{2}\right)$ Volume with 6 Bends}

\subsection{Introduction and Definitions}

Initially, we describe our layout strategy in a static context, in which the entire graph (of maximum degree 6) is available. As will be seen, a dynamic version of the problem follows easily.

Let the vertices of the given graph be $v_{1}, v_{2}, v_{3}, \ldots v_{n}$ (labelled in an arbitrary order). The vertices will be located in a stair-case fashion, separated by an appropriate amount to permit the crossing-free embedding of the edges. More formally, vertex $v_{i}$ is located at $(7 i, 0,5 i)$. For convenience, we denote these coordinates as $\left(X\left(v_{i}\right), Y\left(v_{i}\right), Z\left(v_{i}\right)\right)$. Each vertex may have at most 6 incident edges attached at ports which will be denoted as N, S, E, W, T, B - refer to figure 1 . When viewed from above (i.e. from $+\infty$ along the $\mathrm{Z}$ axis), the compass designations effectively describe the ports.

Containing each vertex $v$ is a small box of dimensions $7 \times 6 \times 5$, called its neighbourhood and denoted by $\mathbf{N}(\mathbf{v})$. Contained entirely inside $\mathrm{N}(\mathrm{v})$ are 6 pedestals - one for each port of $v$. Each pedestal consists of a line segment parallel 

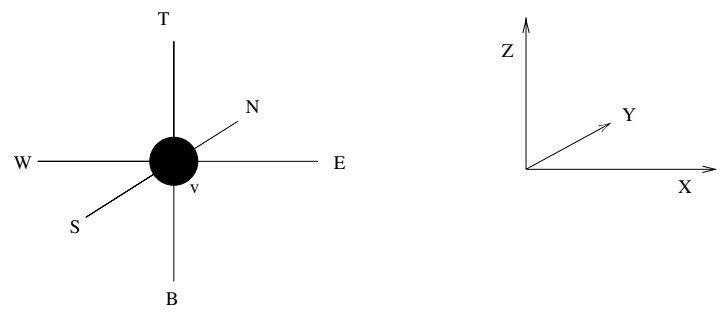

Fig. 1. The 6 ports on a vertex

to the $\mathrm{Z}$ axis. The Bottom pedestal is directly attached to $v$ beneath it, and is of length 1. Similarly, the Top pedestal attaches to the top port of $v$ and is of length 3. The North pedestal is of length 2 and extends from $(X(v),+1, Z(v))$ to $(X(v),+1, Z(v)+2)$. The South pedestal is of length 1 and extends from $(X(v),-1, Z(v))$ to $(X(v),-1, Z(v)+1)$. The West and East pedestals are degenerate points at $(X(v)-1,0, Z(v))$ and $(X(v)+1,0, Z(v))$ respectively; they are introduced for consistency in the description and will be modified in a subsequent section describing a related layout strategy.

The Neighbourhood $\mathrm{N}(\mathrm{v})$ consists of 5 layers in the Z-dimension, the:

- Top layer located on the plane $Z=Z(v)+3$,

- North layer located on the plane $Z=Z(v)+2$,

- South layer located on the plane $Z=Z(v)+1$,

- East, West, $v$ layer located on the plane $Z=Z(v)$,

- Bottom layer located on the plane $Z=Z(v)-1$.

Edges are always considered as directed from the lower indexed vertex to the higher. Each pedestal is used only by outgoing edges from a vertex. Incoming edges are routed along pillars. The 6 pillars of a vertex $v$ extend below the neighbourhood of $v$ to the plane $Z=-1$. More precisely, the

- North pillar extends from $(X(v), 2,-1)$ to $(X(v), 2, Z(v)+2)$,

- South pillar extends from $(X(v),-2,-1)$ to $(X(v),-2, Z(v)+2)$,

- East pillar extends from $(X(v)+2,0,-1)$ to $(X(v)+2,0, Z(v)+2)$,

- West pillar extends from $(X(v)-2,0,-1)$ to $(X(v)-2,0, Z(v)+2)$,

- Bottom pillar extends from $(X(v), 0,-1)$ to $(X(v), 0, Z(v))$,

- Top pillar extends from $(X(v)-3,0,-1)$ to $(X(v)-3,0, Z(v)+3)$.

Note that the four side pillars (N, S, E, W) extend to $Z(v)+2$ to accommodate self loops as will be described in a later section.

We refer to the orthogonal box directly below a vertex $v$ (and containing the pillars of $v$ ), as the airspace of $v$. Edges pass safely through airspaces, by traversing lanes, which are guaranteed to avoid pillars. In particular, note that any line segment lying in the planes $Y=1, Y=3, Y=-1$, or $Y=-3$ intersects no pillar of any vertex.

Typically, an edge from a vertex $v$ to a vertex $w$ leaves $v$ from the specified port, travels to the associated pedestal, climbs the pedestal to the appropriate 


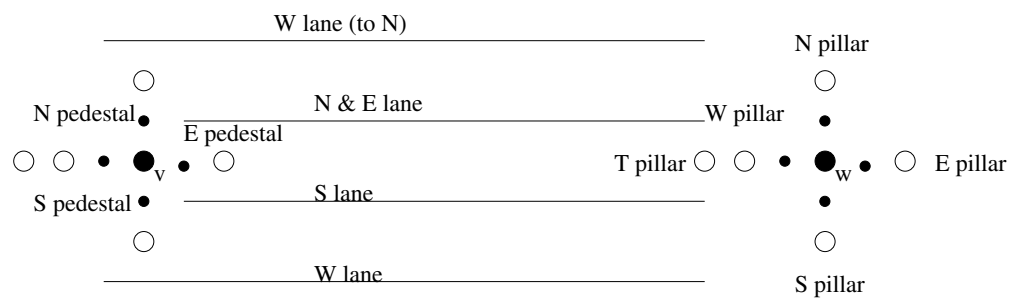

a) View from top
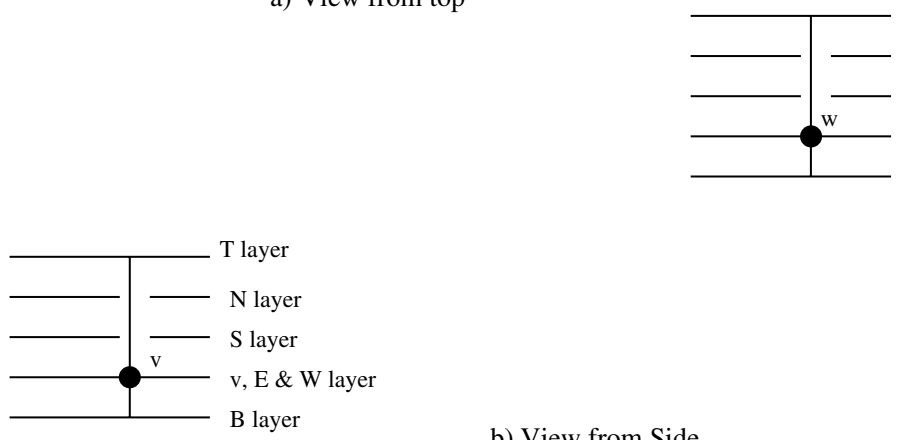

b) View from Side

Fig. 2. Two Neighbourhoods

layer, is routed on that layer to the airspace under $w$ along one of the four lanes, joins to the pillar associated with the destination port of $w$, climbs this pillar and finally enters $w$. Although the routings differ slightly for each port to port pair, this general technique applies, the critical observation being that the routing from the airspace of $v$ to the airspace of $w$ is done at some layer of $v$ (i.e. the lower vertex). By using one of the four lanes, this section of the edge passes safely through the airspace of any intervening vertices without intersecting any of its pillars (which may have edges routed from below). Since the E and W ports of $v$ share the same layer as $v$, special care is required to ensure routings from these ports that do not intersect each other or possible incoming edges to the $\mathrm{N}$ or $\mathrm{S}$ ports. Figure 3 shows the routing of two edges from $v$ to $w$ : a $W \rightarrow N$ connection and a $E \rightarrow E$ connection.

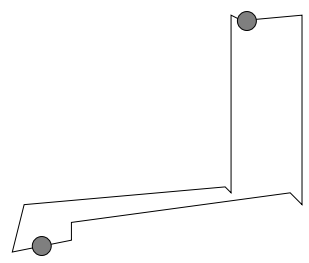

Fig. 3. W to $\mathrm{N}$ and $\mathrm{E}$ to E Routes 
A connection to the Top port of vertex $w$ comes up the Top pillar, along $w$ 's Top layer, and then directly down to $w$, thus safely passing above $w$ 's $\mathrm{W}$ pillar. For completeness, the 36 port-to-port routings are specified in Appendix A. Note that of these routings, 9 require 6 bends, 22 require 5 bends, and 5 require 4 bends.

\subsection{Proof of Correctness}

Lemma 1. No pair of edges in the construction intersect.

Proof:

Appendix A defines the paths for all port-to-port connections between a vertex $v$ (at the lower level) to a vertex $w(\neq v)$. To prove that edge $(v, w)$ intersects no other edge in the layout, note that the routing of each edge consists of three portions:

1. inside $v$ 's neighbourhood

2. along a lane through intervening airspaces

3. in w's airspace.

Since the four lanes intersect no pillars, the second portion of edge $(v, w)$ intersects no other edge.

Consider the portion of the edge $(v, w)$ inside $v$ 's neighbourhood. Each of the 6 possible routings out of $v$ intersect no pillar of $v$ and so can not intersect any incoming edge to $v$. And similarly, the portion in $w$ 's airspace can intersect no outgoing edge from $w$.

It remains to consider the intersection of $(v, w)$ with some outgoing edge from $v$ (inside $v$ 's neighbourhood) or with some other incoming edge to $w$ (inside $w$ 's airspace). Without loss of generality, we consider the case of $(v, w)$ intersecting another edge between $v$ and $w$ but with different port assignments.

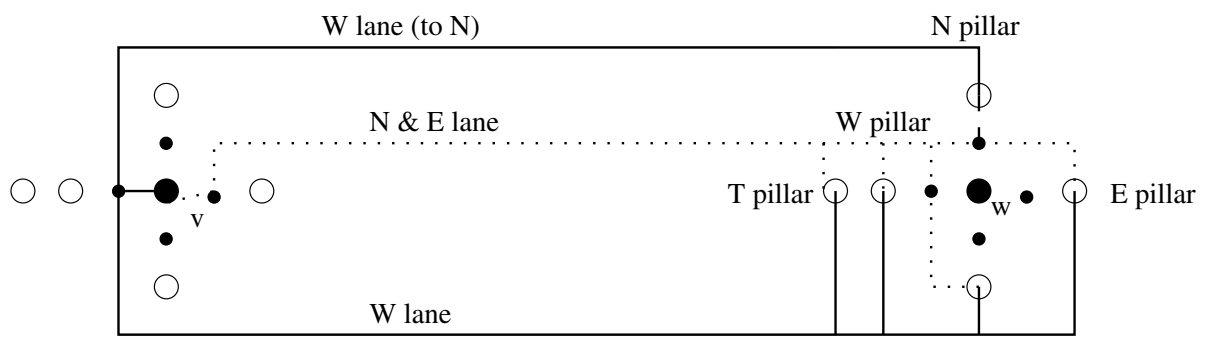

Fig. 4. E and W routings do not intersect

Since the N,S,T, and B ports each have their own planes inside the neighbourhood of $v$, it is simple to verify that no intersections are possible if either of the two edges involve these ports at $v$. However, the $\mathrm{E}$ and $\mathrm{W}$ ports share a plane 
with $v$ itself, and it is therefore critical to check that none of the 30 possible pairs involving these two ports from $v$ intersect. In figure 4 , the 6 routings from $\mathrm{W}$ are shown as solid lines and the 6 routings from $\mathrm{E}$ are displayed as dotted lines; note that only the routings to the pillars in $w$ are shown. That no pair of solid and dotted paths intersect either in the neighbourhood of $v$ nor in the airspace of $w$ is easily determined.

\section{Dynamic Case}

The layout strategy described in the previous section easily adapts to use in a dynamic context, in which both edges and vertices may be deleted or inserted. Insertion of a new vertex $v_{t}$ is performed by placing it at $(7 t, 0,5 t)$. It is clear that a new edge $(u, v)$ can be added to the layout in $O(1)$ time - any free port on $u$ can be connected to a free port on $v$, and furthermore, the overall volume of the layout remains the same. Deletion of a vertex is accomplished by replacing it by the top-most vertex and re-routing the (at most 6 ) incident edges. Let $v_{1}, v_{2}, \ldots v_{t}$ be the vertices in the layout at time $t$. To delete vertex $v_{i}$, the edges on $v_{i}$ are first deleted. Denote by $w_{1}, w_{2}, \ldots w_{6}$ the vertices adjacent to vertex $v_{t}$. These edges are all deleted in the layout and vertex $v_{i}$ is connected to $w_{1}, w_{2}, \ldots w_{6}$, using arbitrary ports on vertex $v_{i}$. Vertex $v_{t}$ is then removed from the layout. The resulting layout has a volume of $6 \cdot 7 \cdot 5(t-1)^{2}$. Note that we measure the number of grid points rather than the length in each dimension.

Theorem 1. Insertion or deletion of vertices or edges can be accomplished in $O(1)$ update time, and at all times, the volume of the layout is $6 \cdot 7 \cdot 5 \cdot t^{2}$, where $t$ is the number of vertices in the layout.

\section{Variants}

In this section, we consider two related constructions - a spiral layout that has a more balanced appearance, and a more elaborate version of the stair-case model that requires at most 5 bends per edge.

\subsection{Achieving 5 Bends per Edge}

In the stair-case layout there are only 9 port-to-port routings that require 6 bends per edge. The layout can be modified to achieve at most 5 bends per edge, however the routings become more involved. The volume bounds and dynamic nature of the algorithm are preserved.

\subsection{Spiral Layout}

One possible criticism of the strategy described in the previous sections is that the dimensions of the resulting layout are "unbalanced" - the construction is very narrow, with a $\mathrm{Y}$ dimension consisting of exactly 7 planes. Although there 
may be practical applications of this feature of our strategy, from an aesthetic standpoint a more balanced layout may be preferable. In this section, we outline a strategy that produces a layout of dimensions $O(\sqrt{n}) \cdot O(\sqrt{n}) \cdot O(n)$, but with a slight penalty on the number of bends: 7 bends are required for some port-to-port connections. The dynamic nature of the previous strategy is preserved; an implementation and a short video describing the routing is available at www.cs.uleth.ca/ ${ }^{\sim}$ wismath/threed.
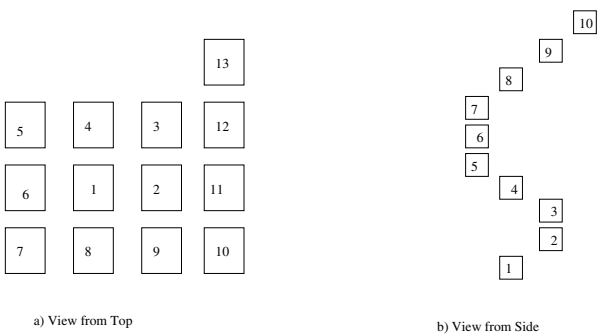

Fig. 5. Spiral layout: a) from above; b) from the side

Instead of placing the vertices in a linear staircase fashion, the vertices are embedded in an orthogonal spiral manner. When viewed from above, the vertices appear in a $\sqrt{n} \cdot \sqrt{n}$ grid as displayed in figure 5 . However, as in the previous method, each vertex is assigned to a unique Z-plane, thus forming a spiral of linear height. Associated with each vertex are 7 planes (one for each port, and one for the vertex itself). The degenerate $\mathrm{E}$ and $\mathrm{W}$ pedestals described in section 2.1 are extended from the $v$ layer to the appropriate plane. The general edge routing technique remains the same. An edge from a vertex $v$ to vertex $w$, exits a port on $v$, climbs a pedestal to the appropriate plane, traverses a lane to correct in the $\mathrm{X}$-coordinate, traverses a lane to correct in the Y-coordinate to enter $w$ 's airspace, climbs the appropriate pillar, and finally enters $w$ at the specified port.

A more detailed account of this spiral layout scheme will be provided in the journal version of the paper.

\subsection{Self-Loops}

The general layout strategy for self loops is similar: from a port to a pedestal, up to the appropriate level, across to the specified pillar, down the pillar and into the vertex. A more detailed description and proof of correctness will be included in the journal version of the paper. A complete listing of the routes is available on the web page.

\section{Implementation and Experimental Results}

One important feature of the layout strategy described in this paper, is its simplicity. An implementation of the layout is available in a package of 3-dimensional 


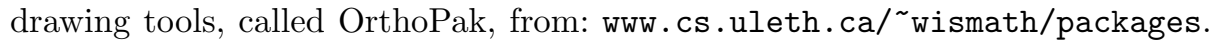
This package was written in $\mathrm{C}++$ and uses the LEDA library extensively. It runs under Solaris 2.6, or linux and produces a VRML world that can be examined with an appropriate browser. OrthoPak is free for research and teaching purposes and is part of a larger suite of research tools developed at the University of Lethbridge.

A test suite of graphs for 3-D orthogonal drawing was described in [3]. Our time trials indicate that our layout strategy is very efficient, 7.013 seconds for the entire test suite of 1820 graphs containing from 5 to 95 vertices running on a SPARC 5. On average, our layouts had between 4.71 and 4.83 bends per edge over the entire distribution of graphs. A more detailed account of the experiments is omitted for lack of space.

\section{Conclusion and Open Problems}

A 3-dimensional orthogonal layout of graphs with $n$ vertices of maximum degree 6 was presented in which each edge is routed with at most 6 bends in a volume bounded by $O\left(n^{2}\right)$. The technique is fully dynamic allowing insertion and deletion of edges and vertices in $O(1)$ time. Multiple edges and self-loops are permitted. An implementation of the drawing strategy is provided, and VRML worlds and a short video describing the technique are also available at: www.cs.uleth.ca/ ${ }^{\sim}$ wismath/threed.

If the layout strategy is not used in a dynamic setting then some preprocessing of the graph is possible and the number of bends can be reduced in the average case. Alternately, special properties of the graph may also be exploitable to reduce the number of bends. For example, trees of maximum degree 6 can be embedded with our construction with no more than 5 bends. An interesting open problem is to determine if there are other classes of graphs that can be routed using this strategy with a minimal amount of pre-processing.

\section{References}

1. T. Biedl, Heuristics for 3D-Orthogonal Graph Drawings, Proceedings of the 4th Twente Workshop on Graphs and Combinatorial Optimization, Enshende, June 1995, pp. 41-44.

2. T. Biedl, T. Shermer, S. Whitesides, S. Wismath, Three-Dimensional Orthogonal Graph Drawing, Symposium on Graph Drawing 97, Lecture Notes in Computer Science 1353, Springer Verlag, 1998, pp. 76-86.

3. G. Di Battista, M. Patrignani, F. Vargiu, A Split \& Push Approach to 3D Orthogonal Drawing, Symposium on Graph Drawing 98, Lecture Notes in Computer Science 1547, Springer Verlag, 1998. pp.87-101.

4. P. Eades, A. Symvonis, S. Whitesides, Two Algorithms for Three-Dimensional Orthogonal Graph Drawing, Symposium on Graph Drawing 96, Lecture Notes in Computer Science 1190, Springer Verlag, 1996, pp. 139-154.

5. A. Papakostas, I. Tollis, Algorithms for Incremental Orthogonal Graph Drawing in Three Dimensions, J. of Graph Algorithms and Applications, to appear. 
6. D. Wood, An Algorithm for Three-Dimensional Orthogonal Graph Drawing, Symposium on Graph Drawing 98, Lecture Notes in Computer Science 1547, Springer Verlag, 1998. pp.332-346.

\section{A Appendix: Routings}

All 36 port-to-port routings from a vertex $v$ to vertex $w$ will now be enumerated. In each case, the initial and terminating points of the route are $(X(v), Y(v)=$ $0, Z(v))$ and $(X(w), Y(w)=0, Z(w))$ respectively.

$-N \rightarrow S:(X(v), 1, Z(v)) \rightarrow(X(v), 1, Z(v)+2) \rightarrow(X(w)-1,1, Z(v)+2) \rightarrow$ $(X(w)-1,-2, Z(v)+2) \rightarrow(X(w),-2, Z(v)+2) \rightarrow(X(w),-2, Z(w))$

$-N \rightarrow N:(X(v), 1, Z(v)) \rightarrow(X(v), 1, Z(v)+2) \rightarrow(X(w), 1, Z(v)+2) \rightarrow$ $(X(w),+2, Z(v)+2) \rightarrow(X(w),+2, Z(w))$

$-N \rightarrow E:(X(v), 1, Z(v)) \rightarrow(X(v), 1, Z(v)+2) \rightarrow(X(w)+2,1, Z(v)+2) \rightarrow$ $(X(w)+2,0, Z(v)+2) \rightarrow(X(w)+2,0, Z(w))$

$-N \rightarrow W:(X(v), 1, Z(v)) \rightarrow(X(v), 1, Z(v)+2) \rightarrow(X(w)-2,1, Z(v)+2) \rightarrow$ $(X(w)-2,0, Z(v)+2) \rightarrow(X(w)-2,0, Z(w))$

$-N \rightarrow T:(X(v), 1, Z(v)) \rightarrow(X(v), 1, Z(v)+2) \rightarrow(X(w)-3,1, Z(v)+2) \rightarrow$ $(X(w)-3,0, Z(v)+2) \rightarrow(X(w)-3,0, Z(w)+3) \rightarrow(X(w), 0, Z(w)+3)$

$-N \rightarrow B:(X(v), 1, Z(v)) \rightarrow(X(v), 1, Z(v)+2) \rightarrow(X(w), 1, Z(v)+2) \rightarrow$ $(X(w), 0, Z(v)+2)$

$-S \rightarrow N:(X(v),-1, Z(v)) \rightarrow(X(v),-1, Z(v)+1) \rightarrow(X(w)-1,-1, Z(v)+$ $1) \rightarrow(X(w)-1,2, Z(v)+1) \rightarrow(X(w), 2, Z(v)+1) \rightarrow(X(w), 2, Z(w))$

$-S \rightarrow S:(X(v),-1, Z(v)) \rightarrow(X(v),-1, Z(v)+1) \rightarrow(X(w),-1, Z(v)+1) \rightarrow$ $(X(w),-2, Z(v)+1) \rightarrow(X(w),-2, Z(w))$

$-S \rightarrow E:(X(v),-1, Z(v)) \rightarrow(X(v),-1, Z(v)+1) \rightarrow(X(w)+2,-1, Z(v)+$ $1) \rightarrow(X(w)+2,0, Z(v)+1) \rightarrow(X(w)+2,0, Z(w))$

$-S \rightarrow W:(X(v),-1, Z(v)) \rightarrow(X(v),-1, Z(v)+1) \rightarrow(X(w)-2,-1, Z(v)+$ $1) \rightarrow(X(w)-2,0, Z(v)+1) \rightarrow(X(w)-2,0, Z(w))$

$-S \rightarrow T:(X(v),-1, Z(v)) \rightarrow(X(v),-1, Z(v)+1) \rightarrow(X(w)-3,-1, Z(v)+$ $1) \rightarrow(X(w)-3,0, Z(v)+1) \rightarrow(X(w)-3,0, Z(w)+3) \rightarrow(X(w), 0, Z(w)+3)$

$-S \rightarrow B:(X(v),-1, Z(v)) \rightarrow(X(v),-1, Z(v)+1) \rightarrow(X(w),-1, Z(v)+1) \rightarrow$ $(X(w), 0, Z(v)+1)$

$-E \rightarrow N:(X(v)+1,0, Z(v)) \rightarrow(X(v)+1,1, Z(v)) \rightarrow(X(w), 1, Z(v)) \rightarrow$ $(X(w), 2, Z(v)) \rightarrow(X(w), 2, Z(w))$

$-E \rightarrow S:(X(v)+1,0, Z(v)) \rightarrow(X(v)+1,1, Z(v)) \rightarrow(X(w)-1,1, Z(v)) \rightarrow$ $(X(w)-1,-2, Z(v)) \rightarrow(X(w),-2, Z(v)) \rightarrow(X(w),-2, Z(w))$

$-E \rightarrow E:(X(v)+1,0, Z(v)) \rightarrow(X(v)+1,1, Z(v)) \rightarrow(X(w)+2,1, Z(v)) \rightarrow$ $(X(w)+2,0, Z(v)) \rightarrow(X(w)+2,0, Z(w))$

$-E \rightarrow W:(X(v)+1,0, Z(v)) \rightarrow(X(v)+1,1, Z(v)) \rightarrow(X(w)-2,1, Z(v)) \rightarrow$ $(X(w)-2,0, Z(v)) \rightarrow(X(w)-2,0, Z(w))$

$-E \rightarrow T:(X(v)+1,0, Z(v)) \rightarrow(X(v)+1,1, Z(v)) \rightarrow(X(w)-3,1, Z(v)) \rightarrow$ $(X(w)-3,0, Z(v)) \rightarrow(X(w)-3,0, Z(w)+3) \rightarrow(X(w), 0, Z(w)+3)$

$-E \rightarrow B:(X(v)+1,0, Z(v)) \rightarrow(X(v)+1,1, Z(v)) \rightarrow(X(w), 1, Z(v)) \rightarrow$ $(X(w), 0, Z(v))$ 
$-W \rightarrow N:(X(v)-1,0, Z(v)) \rightarrow(X(v)-1,3, Z(v)) \rightarrow(X(w), 3, Z(v)) \rightarrow$ $(X(w), 2, Z(v)) \rightarrow(X(w), 2, Z(w))$

$-W \rightarrow S:(X(v)-1,0, Z(v)) \rightarrow(X(v)-1,-3, Z(v)) \rightarrow(X(w),-3, Z(v)) \rightarrow$ $(X(w),-2, Z(v)) \rightarrow(X(w),-2, Z(w))$

$-W \rightarrow W:(X(v)-1,0, Z(v)) \rightarrow(X(v)-1,-3, Z(v)) \rightarrow(X(w)-2,-3, Z(v)) \rightarrow$ $(X(w)-2,0, Z(v)) \rightarrow(X(w)-2,0, Z(w))$

$-W \rightarrow E:(X(v)-1,0, Z(v)) \rightarrow(X(v)-1,-3, Z(v)) \rightarrow(X(w)+2,-3, Z(v)) \rightarrow$ $(X(w)+2,0, Z(v)) \rightarrow(X(w)+2,0, Z(w))$

$-W \rightarrow T:(X(v)-1,0, Z(v)) \rightarrow(X(v)-1,-3, Z(v)) \rightarrow(X(w)-3,-3, Z(v)) \rightarrow$ $(X(w)-3,0, Z(v)) \rightarrow(X(w)-3,0, Z(w)+3) \rightarrow(X(w), 0, Z(w)+3)$

$-W \rightarrow B:(X(v)-1,0, Z(v)) \rightarrow(X(v)-1,-3, Z(v)) \rightarrow(X(w)+1,-3, Z(v)) \rightarrow$ $(X(w)+1,0, Z(v)) \rightarrow(X(w), 0, Z(v))$

$-T \rightarrow N:(X(v), 0, Z(v)+3) \rightarrow(X(v),+1, Z(v)+3) \rightarrow(X(w), 1, Z(v)+3) \rightarrow$ $(X(w), 2, Z(v)+3) \rightarrow(X(w), 2, Z(w))$

$-T \rightarrow S:(X(v), 0, Z(v)+3) \rightarrow(X(v),-1, Z(v)+3) \rightarrow(X(w),-1, Z(v)+3) \rightarrow$ $(X(w),-2, Z(v)+3) \rightarrow(X(w),-2, Z(w))$

$-T \rightarrow E:(X(v), 0, Z(v)+3) \rightarrow(X(v),+1, Z(v)+3) \rightarrow(X(w)+2,1, Z(v)+$ $3) \rightarrow(X(w)+2,0, Z(v)+3) \rightarrow(X(w)+2,0, Z(w))$

$-T \rightarrow W:(X(v), 0, Z(v)+3) \rightarrow(X(v),-1, Z(v)+3) \rightarrow(X(w)-2,-1, Z(v)+$ $3) \rightarrow(X(w)-2,0, Z(v)+3) \rightarrow(X(w)-2,0, Z(w))$

$-T \rightarrow B:(X(v), 0, Z(v)+3) \rightarrow(X(v),-1, Z(v)+3) \rightarrow(X(w),-1, Z(v)+3) \rightarrow$ $(X(w), 0, Z(v)+3)$

$-T \rightarrow T:(X(v), 0, Z(v)+3) \rightarrow(X(v),+1, Z(v)+3) \rightarrow(X(w)-3,1, Z(v)+$ $3) \rightarrow(X(w)-3,0, Z(v)+3) \rightarrow(X(w)-3,0, Z(w)+3) \rightarrow(X(w), 0, Z(w)+3)$

$-B \rightarrow N:(X(v), 0, Z(v)-1) \rightarrow(X(v),+1, Z(v)-1) \rightarrow(X(w), 1, Z(v)-1) \rightarrow$ $(X(w), 2, Z(v)-1) \rightarrow(X(w), 2, Z(w))$

$-B \rightarrow S:(X(v), 0, Z(v)-1) \rightarrow(X(v),-1, Z(v)-1) \rightarrow(X(w),-1, Z(v)-1) \rightarrow$ $(X(w),-2, Z(v)-1) \rightarrow(X(w),-2, Z(w))$

$-B \rightarrow E:(X(v), 0, Z(v)-1) \rightarrow(X(v),+1, Z(v)-1) \rightarrow(X(w)+2,1, Z(v)-$ $1) \rightarrow(X(w)+2,0, Z(v)-1) \rightarrow(X(w)+2,0, Z(w))$

$-B \rightarrow W:(X(v), 0, Z(v)-1) \rightarrow(X(v),-1, Z(v)-1) \rightarrow(X(w)-2,-1, Z(v)-$ $1) \rightarrow(X(w)-2,0, Z(v)-1) \rightarrow(X(w)-2,0, Z(w))$

$-B \rightarrow T:(X(v), 0, Z(v)-1) \rightarrow(X(v),+1, Z(v)-1) \rightarrow(X(w)-3,1, Z(v)-$ $1) \rightarrow(X(w)-3,0, Z(v)-1) \rightarrow(X(w)-3,0, Z(w)+3) \rightarrow(X(w), 0, Z(w)+3)$

$-B \rightarrow B:(X(v), 0, Z(v)-1) \rightarrow(X(v),-1, Z(v)-1) \rightarrow(X(w),-1, Z(v)-1) \rightarrow$ $(X(w), 0, Z(v)-1)$ 